\title{
Heterogeneity formation within biofilm systems $\dagger$
}

\author{
ANDREAS C. ARISTOTELOUS 1 , YURY GRABOVSKY² and \\ ISAAC KLAPPER ${ }^{2}$ \\ ${ }^{1}$ Department of Mathematics, West Chester University, West Chester, PA, USA \\ ${ }^{2}$ Department of Mathematics, Temple University, Philadelphia, PA, USA \\ email:klapper@temple.edu
}

(Received 11 July 2017; revised 9 June 2018; accepted 11 June 2018; first published online 16 July 2018)

\begin{abstract}
Biofilms, and collections of embedded microbial communities, present structural heterogeneities with functional consequences for important processes, such as transport. The origin of such structures has been unclear. Here, we propose that they can arise as a consequence of diffusive transport limitation. To illustrate, a model allowing internal heterogeneity is developed. Linear analysis is applied to a simplified version of the model suggesting that heterogeneity forms on (or below) the active layer length, a length scale that may not be suitable for homogenization, with non-trivial implications for system scale properties such as reduction in system-wide diffusive transport efficiency. Numerics suggest that the simplified model provides useful insight into behaviour of the full model. We then show examples based on microcolony formation in host domains and argue that internal heterogeneity can be related to community function.
\end{abstract}

Key words: Biofilms, heterogeneity, diffusive transport.

\section{Introduction}

The label 'biofilm' is something of a misnomer as biofilms are generally structurally heterogeneous, both transversely and horizontally, or maybe even occurring as inclusions in larger structures, see Figure 1, and often are not necessarily well described by the usual mathematical connotation of the term film. Transverse structure is a hallmark of biofilm function, and as such, has received considerable attention both in observational and modeling studies [22,27,39]. Heterogeneity in horizontal structure has also been documented, though with less attention $[22,32]$. The mechanisms that result in such heterogeneity in biofilm systems are unclear.

Here, we propose a physical mechanism that can generate internal heterogeneity in biomaterial density; hotspots of locally enhanced biomaterial concentrations cause locally increased substrate sinks, and hence lead to locally increased substrate transport (Figure 2), and at the same time, local depletion of substrate concentration. With increased substrate flux, these biomaterial hotspots can exhibit relatively enhanced growth at the expense of their nearby neighbours resulting in internal heterogeneity. As this mechanism is diffusive

$\dagger$ The authors acknowledge funding provided for this project by NSF Award Nos. 1517100 and 1720226, and NIH Award No. R01GM109452. 

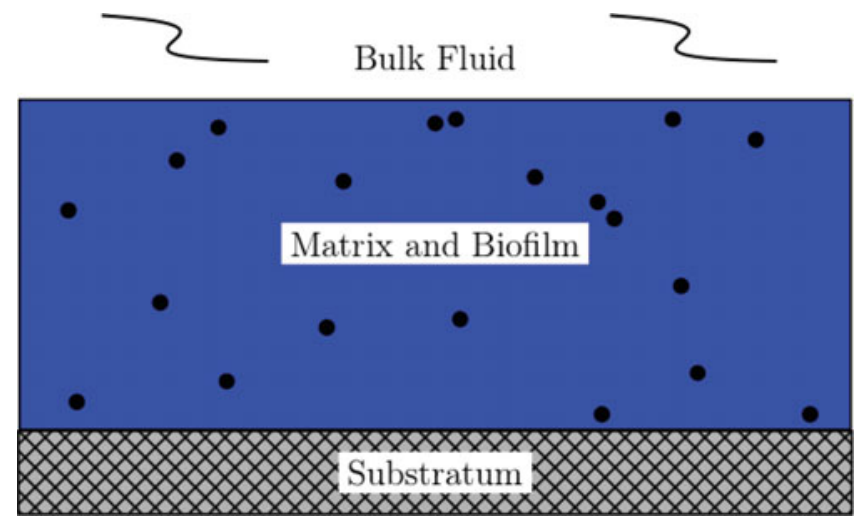

FIGURE 1. Internal micro-biofilms (black circles) included in a larger matrix.

transport based, it is most apparent at the active layer length scale

$$
\lambda_{G}=\sqrt{\frac{D}{r}},
$$

where $D$ is a substrate diffusivity and $r$ is a substrate usage rate. This length is often associated with the presence of increased metabolic activity, typically near the biofilmbulk fluid interface [39]. Roughly, $\lambda_{G}$ measures the length over which substrate can diffuse before being significantly depleted by usage. At longer lengths, diffusion is unable to maintain hotspot-generated gradients in substrate concentration.

The length $\lambda_{G}$ is a significant one as it also has been argued to be the material homogenizability length scale for heterogeneous biofilm systems [5]. Heterogeneities on smaller lengths can be regarded as mathematically homogenizable with respect to diffusive transport, while heterogeneities on larger lengths cannot; this begs the dynamical question which we address here: would a homogeneous, growing system be stable to internal perturbations and, if not, at what length scale would growth-driven heterogeneities emerge? Interestingly, the answer predicted below is that, in fact, internal instabilities arise at roughly this same length scale $\lambda_{G}$. That is, we argue that physical considerations lead to formation of structures on the same length scale as the one that separates homogenizable from non-homogenizable systems.

These remarks apply not only to heterogeneity within biofilms themselves but also to mixtures of microbial colonies embedded in matrices of materials of various kinds, for example, colonies within chronic wounds and cystic fibrosis related mucus plugs [9,24,37] and, recently, construction of synthetic communities through $3 \mathrm{D}$ printing techniques [29, 35]. But, within-biofilm heterogeneity can also be important. Community function itself is likely influenced by internal heterogeneity, for example, [30,44]. Ecology in such systems is thus also likely coupled to structure and is almost certainly linked to diffusive transport, so that biomaterial heterogeneity may be of importance to long time dynamics, though, we do not consider such things here as a more complicated model would be necessary. Unsurprisingly, mechanical properties are effected by internal heterogeneity as well [16], also beyond our scope here, but modeled elsewhere, for example [15,26]. 

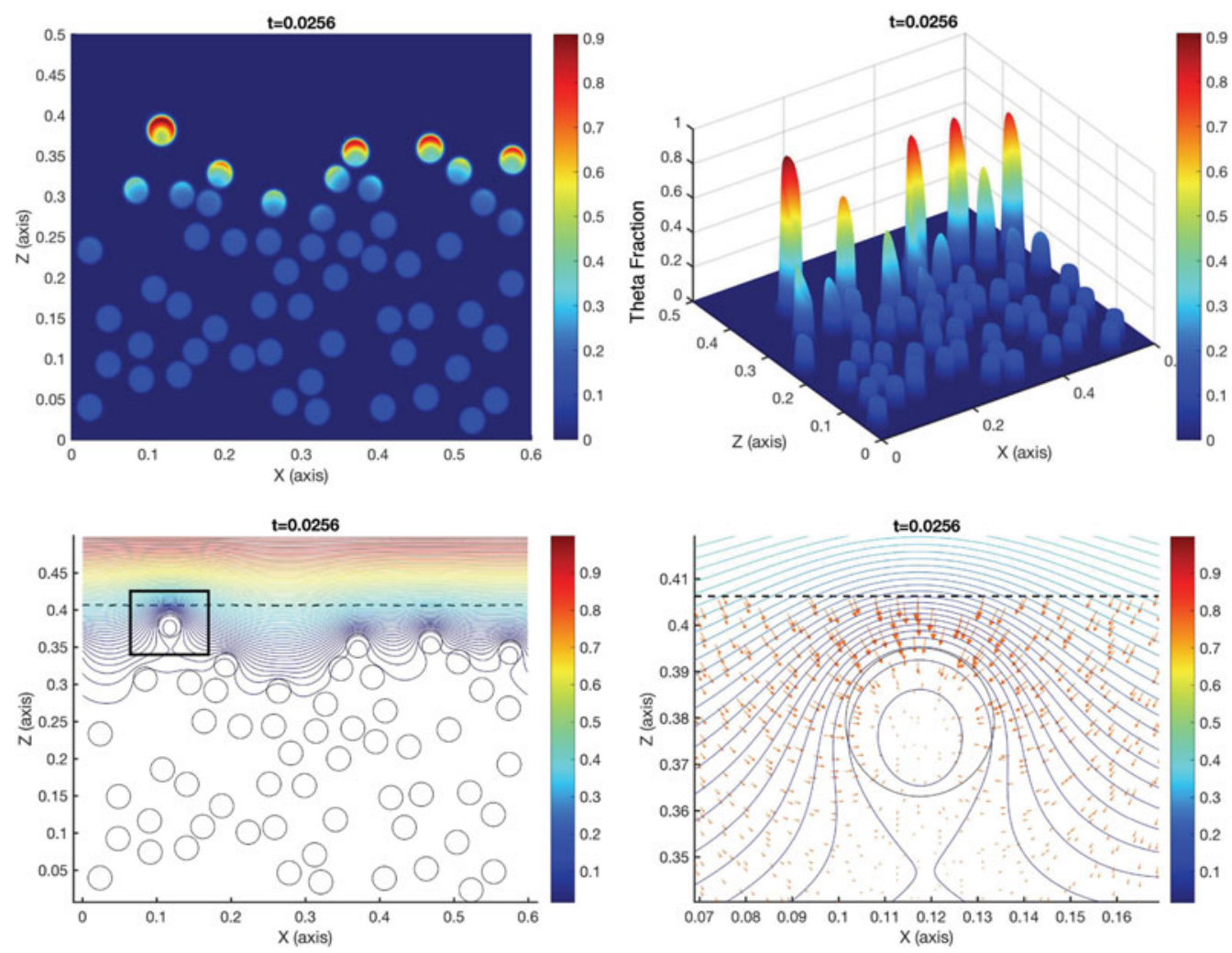

FIGURE 2. Simulation of biomaterial microcolonies (with volume fraction $\theta$ ) inclusions in a broader matrix material. Top: biomaterial. Colour is related to biomass volume fraction, which in turn is related to biomass activity. Bottom: substrate. Contours of substrate concentration, superimposed on the microcolony locations (circles), shown on the left. Dashed line is the interface between biofilm-matrix and pure diffusive subregions. Inset square is blown up on the right together with arrows indicating the direction and relative amplitude of diffusive transport. Note that microcolonies nearer to the top of the box have improved access to substrate diffusing in from the top $z=0.5$, resulting in increased biomass, and thus, in enhanced substrate sinks capable of depleting substrate from their neighbourhood. It is this local depletion of substrate which is proposed here to be responsible for structure formation. Details of model and computational methods provided below.

Relatedly, a number of modeling studies, for example, $[15,18,33]$, have suggested that a different type of structure, that of finger formation, may also be a consequence of diffusive transport limitation. In particular, a flat biofilm layer is typically susceptible to an expansion driven instability, in which small bumps protruding towards an exterior substrate source are able to gain improved access to that source and hence grow into, eventually, finger-like structures. These fingers exhibit a characteristic length scale, the same active length $\lambda_{G}$. This scale emerges in both fingering and heterogeneity phenomenon because it provides a length scale over which protrusions can 'steal' substrate from the environment. That is, the unstable fingering length scale is also $\mathrm{O}\left(\lambda_{G}\right)$. However, the details of the driving instability for fingering are different than the ones described here in that, in the fingering case, increased substrate transport is driven chiefly by decreasing transport 
distance rather than increasing the strength of the substrate sink. Thus, the fingering instability is related to the one presented here but the underlying mechanism for gradient enhancement is different in important respects.

The paper is organized as follows. First, we introduce a growth model based on diffusion-reaction physics for embedded biomaterial and then employ the mathematical artifice of perturbing a supposed, already present homogeneous system. A linearization argument for this simplified system suggests that it is unstable at length scale $\lambda_{G}$ or smaller, from which we infer as a consequence that (a) homogeneous systems should not be observed, and (b) heterogeneity may exhibit a signature length scale. We use numerical simulations of the full model applied to perturbed homogeneous initial conditions in order to argue that the results of the linearization are valid, despite the simplification. Of course, perturbation of a homogeneous state is somewhat artificial and is meant instead to argue, here, that a homogeneous state should not exist and rather a heterogeneous state with a predicted length scale should be observed. Hence, we also present simulations of strongly heterogeneous systems reminiscent of microbial colonies embedded in a background matrix (e.g., a mucus layer) in order to demonstrate possible effects of heterogeneity in the strongly non-linear regime.

\section{Model description}

The model domain consists of a slab geometry with two compartments: a biofilm and matrix region $0<z<H$ and a diffusive layer $H<z<H+L$. We introduce a biomaterial volume fraction $\theta(\mathbf{x}, t)$ with $0 \leqslant \theta \leqslant 1$ for $0<z<H$ and $\theta=0$ for $H<z<H+L$. Substrate is described by concentration function $c(\mathbf{x}, t)$. Equations for $c$ and $\theta$ take the form

$$
\begin{aligned}
\nabla \cdot(D(z) \nabla c) & =r(c) \theta, \\
\frac{\partial}{\partial t} \theta & =\operatorname{Yr}(c) f(\theta),
\end{aligned}
$$

where $r(c)$ is a reaction function (substrate/time) and $f(\theta)$ (unitless) is a population growth activity. In addition, $Y$ is a yield coefficient (volume fraction/substrate) and

$$
D(z)= \begin{cases}D^{+}, & H<z<H+L, \\ D^{-}, & 0<z<H,\end{cases}
$$

is a piecewise constant diffusivity. Equation (2.1) is accompanied by boundary conditions $\left.c_{z}\right|_{z=0}=0,\left.c\right|_{z=H+L}=c_{0}$, where $c_{0}>0$ provides a substrate supply, and by interface conditions $[c]=\left[D c_{z}\right]=0$, where [.] denotes jump across the $z=H$ interface between biofilm-matrix and diffusive layer regions. Equation (2.2) is supplied with initial conditions $\theta(\mathbf{x}, 0)$. For reaction function $r(c)$ we use either first order (linear) form $r(c)=r_{0} c$ or Monod form $r(c)=r_{\max } c /(K+c)$. For population growth activity, we use either $f(\theta)=\theta$ or $f(\theta)=\theta(1-\theta)$. The latter choice prevents volume fraction from increasing beyond $\theta=1$, though for the most part we avoid the $\theta \approx 1$ regime anyway, in which case we don't observe significant differences between the two. Note that equation (2.2) differs from the typical material law used in biofilm models, for example, $[15,18,19]$, where some sort 
of growth-driven pressure causes material expansion and deformation. Here, rather, we view microbes as embedded in a background matrix where new biomaterial essentially slips through the background matrix.

Note that the substrate reaction-diffusion equation (2.1) is assumed to be at quasiequilibrium, that is, the time derivative term $c_{t}$ is neglected as we suppose that the domain is thin enough so that the time-scale for reaction-diffusion processes to equilibrate is significantly shorter than the growth time scale. This assumption is common in biofilm models [27]. Also, we will assume for simplicity in computations that $D$ is constant, that is, $D^{+}=D^{-}$.

\section{1D solution}

If we suppose the initial conditions to be dependent on $z$ only, that is, $\theta(\mathbf{x}, 0)=\theta(z, 0)$, then system (2.1)-(2.2) becomes one dimensional. That is, $c=c(z, t), \theta=\theta(z, t)$, where

$$
\begin{aligned}
\left(D(z) c_{z}\right)_{z} & =r(c) \theta, \\
\frac{\partial}{\partial t} \theta & =\operatorname{Yr}(c) f(\theta),
\end{aligned}
$$

on the domain $0<z<H+L$ with boundary conditions $c_{z}(0, t)=0, c(H+L, t)=c_{0}$, and interface conditions $[c]=\left[D c_{z}\right]=0$ at $z=H$. For reasonable assumptions on the forms of $r$ and $f$ and initial conditions $\theta(z, 0)$, equation (3.1) has a unique, monotone increasing solution (and a similar statement holds for the initial value problem (3.2)). Note that in the diffusive layer $H<z<H+L$, the substrate equation reduces to $c_{z z}=0$ with $c(H+L)=c_{0}$, which has solution that, when combined with the interface conditions, allows restriction of (3.1)-(3.2) to the biofilm-matrix region $0<z<H$, except now with boundary conditions $c_{z}(0, t)=0$ and $c(H, t)+\left(D^{-} / D^{+}\right) L c_{z}(H, t)=c_{0}$.

See Figure 3 for a typical solution of (3.1)-(3.2), numerical methods described later. Note the formation of an active layer $\lambda_{G}=\sqrt{D / r_{\max }} \approx 0.01$. (In fact, we should use a modified definition $\lambda_{G}=\sqrt{D / r_{\max } \bar{\theta}}$, where $\bar{\theta}$ is a typical volume fraction, but $\theta$ rapidly approaches 1 in the relevant region of Figure 3, top middle.) Note also that as biomaterial increases, net flux of substrate into the biofilm-matrix region increases as well and in fact approaches an asymptote [25].

\section{Linear theory}

Equations (2.1) and (2.2) do not have closed form solutions, generally, even for choices $r(c)=r_{0} c$ and $f(\theta)=\theta$. Nevertheless, it is useful to consider a linear theory based on perturbation of a spatially homogeneous biomaterial volume fraction $\theta(\mathbf{x}, t)=\theta_{0}(t)$. In particular, we replace equation (2.2) by a simplified version

$$
\frac{\partial}{\partial t} \theta(x, t)=\operatorname{Yr}(c(x, H, t)) f(\theta(x, t))
$$

with depth independent substrate concentration. The resulting dispersion relation, of course, is not the correct one for system (2.1)-(2.2); but, we can hope that it is qualitatively similar due to the fact that overall growth of biomaterial is dominated by the growth 

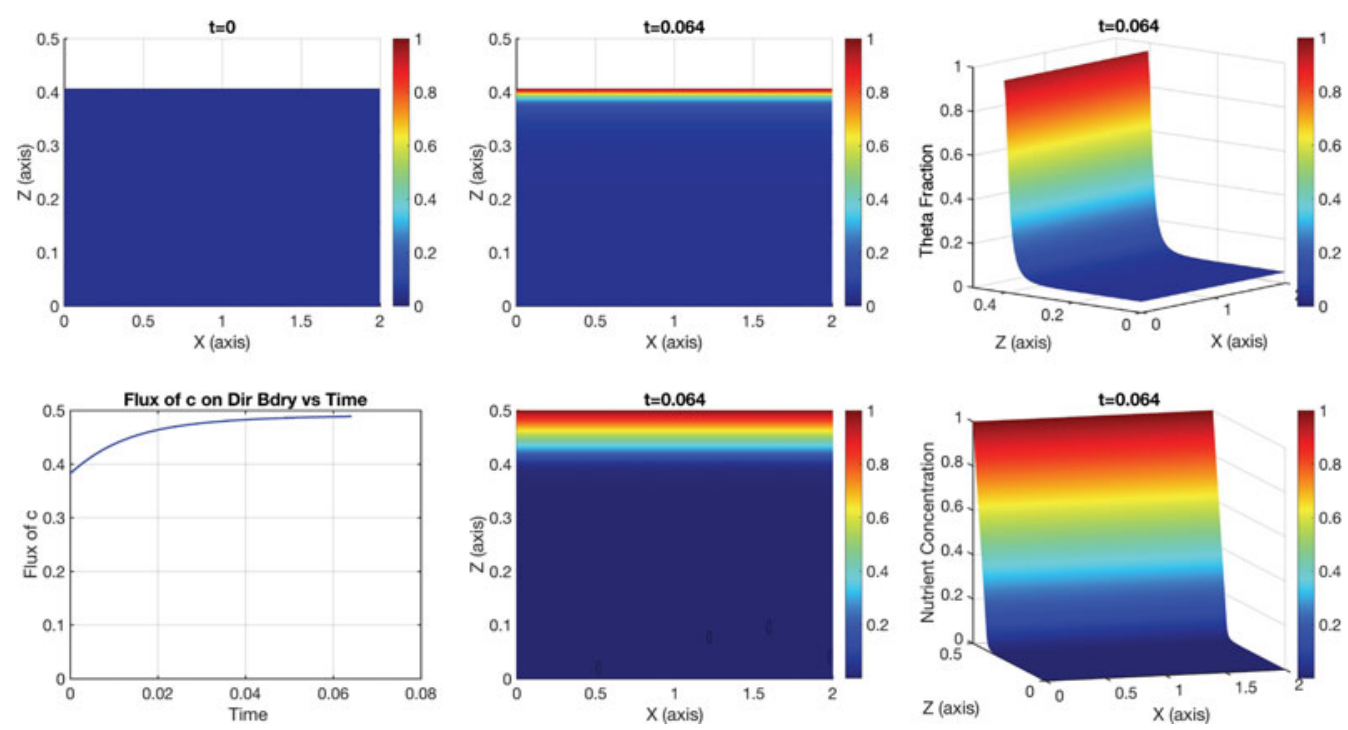

FIGURE 3. Example solution of (3.1)-(3.2). Top row: volume fraction $\theta(z, t)$ with initial conditions (top left) $\theta(z, 0)=0.05$. Bottom left: flux of $c$ through the top of the biofilm-matrix region. Bottom middle, right: concentration $c(z, t)$. Parameters: $r_{\max }=720, K=0.8, Y=1, D^{+}=D^{-}=0.05$, $c_{0}=1, H=0.40625, H+L=0.5$.

in the active layer, where in comparison to the entire biofilm-matrix layer, the substrate concentration $c$ is approximately equal to $c(x, H, t)$. Setting $c=c(x, H, t)$ is in fact an overestimate of substrate concentration, and hence, can be expected to lead to overestimates of growth rate. Afterwards, we compare results of this simplified perturbation analysis to numerical solution of the full system to check qualitative conclusions.

We set $r(c)=r_{0} c$ and $f(\theta)=\theta$. We remark that choice of linear kinetics for $r$, rather than, say, Monod kinetics, eases computation, while not changing net substrate flux significantly [25]; so, we do not expect significant impact on qualitative results. Now, assuming $\theta(\mathbf{x}, 0)=0$ for $z>H$, equation (2.1) can be written as

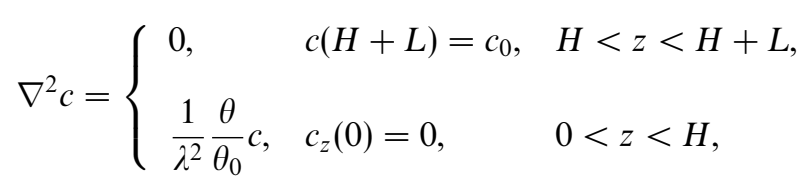

with $[c]=\left[D c_{z}\right]=0$ at the interface $z=H$. The length scale $\lambda=\sqrt{D^{-} / r_{0} \theta_{0}}$ is the effective active layer depth. The quantity $\theta_{0}$, here, is an arbitrary representative value, but in the case that $\theta$ is independent of $z$, that is, $\theta=\theta_{0}$, equation (4.2) has solution

$$
C(z)=\left\{\begin{array}{ll}
c_{0} A(H+L-z)+c_{0} & H<z<H+L \\
c_{0} B \frac{\cosh (z / \lambda)}{\cosh (H / \lambda)} & 0<z<H
\end{array},\right.
$$


where

$$
\begin{aligned}
& A=-\frac{1}{L} \frac{\frac{D^{-}}{D^{+}} \frac{L}{\lambda} \tanh \left(\frac{H}{\lambda}\right)}{1+\frac{D^{-}}{D^{+}} \frac{L}{\lambda} \tanh \left(\frac{H}{\lambda}\right)}, \\
& B=\frac{1}{1+\frac{D^{-}}{D^{+}} \frac{L}{\lambda} \tanh \left(\frac{H}{\lambda}\right)} .
\end{aligned}
$$

Equation (2.2) is replaced by the simplified version (4.1) so that

$$
\frac{\partial}{\partial t} \theta=\frac{d}{d t} \theta_{0}=\operatorname{Yr}\left(\left.c\right|_{z=H}\right) f\left(\theta_{0}\right)=Y r_{0} C(H) \theta_{0}=Y r_{0} c_{0} B \theta_{0}
$$

and hence

$$
\theta(t)=\theta_{0}(t)=\theta_{0}(0) e^{Y r_{0} c_{0} B t} .
$$

Next, in order to study instability, we suppose a spatially sinusoidal (in $x$ ) perturturbation to an otherwise homogeneous initial biomaterial volume fraction and linearize. We write fields $c(\mathbf{x}, t)$ and $\theta(\mathbf{x}, t)$ as

$$
\begin{aligned}
& c(\mathbf{x}, t)=C(z, t)+\tilde{c}(z, t) \sin (x / \ell), \\
& \theta(\mathbf{x}, t)=\theta_{0}(t)+\tilde{\theta}(t) \sin (x / \ell) .
\end{aligned}
$$

In the diffusive layer, $\tilde{c}$ satisfies

with $\tilde{c}(H+L)=0$, so that

$$
\frac{d^{2}}{d z^{2}} \tilde{c}-\frac{1}{\ell^{2}} \tilde{c}=0
$$

$$
\tilde{c}(z, t)=E c_{0} \sinh \left(\frac{H+L-z}{\ell}\right), \quad H<z<H+L,
$$

for a constant $E$. In the biofilm-matrix region, $\tilde{c}$ satisfies

$$
\frac{d^{2}}{d z^{2}} \tilde{c}-\frac{1}{\Lambda^{2}} \tilde{c}=\frac{1}{\lambda^{2}} \frac{\tilde{\theta}}{\theta_{0}} C
$$

with $(d / d z) \tilde{c}(0)=0$, where

Solving, we obtain

$$
\frac{1}{\Lambda^{2}}=\frac{1}{\lambda^{2}}+\frac{1}{\ell^{2}}
$$

$$
\tilde{c}(z, t)=F c_{0} B \frac{\cosh \left(\frac{z}{\Lambda}\right)}{\cosh \left(\frac{H}{\Lambda}\right)}-\frac{\ell^{2}}{\lambda^{2}} \frac{\tilde{\theta}}{\theta_{0}} c_{0} B \frac{\cosh \left(\frac{z}{\lambda}\right)}{\cosh \left(\frac{H}{\lambda}\right)}, \quad 0<z<H .
$$

Coefficients $E$ and $F$ are determined by interface conditions for $\tilde{c}$. In particular,

$$
F=\frac{\ell^{2}}{\lambda^{2}} \frac{\tilde{\theta}}{\theta_{0}} \frac{1+\frac{D^{-}}{D^{+}} \frac{\ell}{\lambda} \tanh \left(\frac{L}{\ell}\right) \tanh \left(\frac{H}{\lambda}\right)}{1+\frac{D^{-}}{D^{+}} \frac{\ell}{\Lambda} \tanh \left(\frac{L}{\ell}\right) \tanh \left(\frac{H}{\Lambda}\right)} .
$$




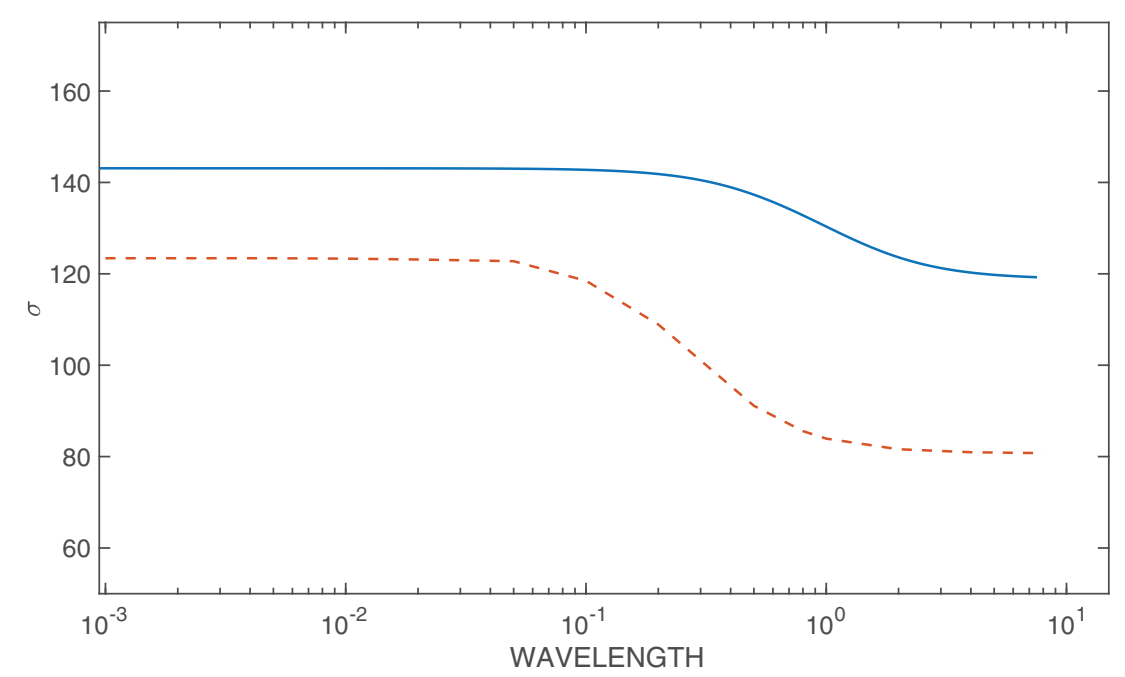

FIGURE 4. Solid curve is the dispersion coefficient $\sigma$ versus $\ell$ for the simplified model (see (4.4)) and dashed curve is the corresponding coefficient for the full model computed numerically. In both cases, $\lambda_{t=0}=0.05$, approximately the 'edge' of the faster growth plateau. Because the simplified model has increased biomass, its effective active layer is smaller (shifting the dispersion curve to the right) and growth is somewhat faster since effective flux of substrate into the biofilm layer is larger (shifting the dispersion curve upwards). Parameter values: $Y=1, r_{0}=400, c_{0}=1, D^{-}=D^{+}=0.05$, $H=0.40625, L=0.09375, \theta_{0}(0)=0.05$.

The perturbed biomaterial volume fraction $\tilde{\theta}$ satisfies the linearized equation

$$
\frac{\partial}{\partial t} \tilde{\theta}(z, t)=Y r_{0} C(H, t) \tilde{\theta}(z, t)+Y r_{0} \tilde{c}(H, t) \theta_{0}(t)=\sigma \tilde{\theta}(z, t)
$$

where

$$
\sigma(\ell)=Y r_{0} c_{0} B\left(1+\frac{\ell^{2}}{\lambda^{2}} \frac{1+\frac{D^{-}}{D^{+}} \frac{\ell}{\lambda} \tanh \left(\frac{L}{\ell}\right) \tanh \left(\frac{H}{\lambda}\right)}{1+\frac{D^{-}}{D^{+}} \frac{\ell}{\Lambda} \tanh \left(\frac{L}{\ell}\right) \tanh \left(\frac{H}{\Lambda}\right)}-\frac{\ell^{2}}{\lambda^{2}}\right)
$$

is the dispersion relation. In Figure 4, we plot $\sigma(\ell)$ for the simplified model, as given by (4.4), versus $\sigma(\ell)$ computed numerically for the full model. For the numerics, we apply initial conditions $\theta(0)=0.05(1+0.001 \cos (2 \pi s / \ell))$ and then estimate $\sigma(\ell)$ from the rate of increase in the gap between the maximum and minimum values of $\left.\theta(t)\right|_{z=H}$. Computations use the full model (2.1)-(2.2) with $f(\theta)=\theta$ and methods as described below. While the simplified $\sigma$ differs from the (numerically approximated) true one, they have qualitative similarities. In particular, note the smoothed step function profile, with the step occurring where perturbation length scale $\ell$ and active length scale $\lambda_{G}$ are comparable. The step plateaus indicate two basic features of the dynamics. First, there is a base level of growth that is independent of perturbation length scale $\ell$ (see the first term $Y r_{0} C(H, t) \tilde{\theta}(z, t)$ in (4.3)) arising from the fact that, independent of neighbours, locally increased biomaterial depletes substrate locally, and hence, increases influx. Second, there is an enhanced level of growth at perturbation length scales smaller, approximately, than the active length (see the second term $Y r_{0} \tilde{c}(H, t) \theta_{0}(t)$ in (4.3)) arising from the fact that if regions of increased and decreased biomaterial are sufficiently close, then, the increased 
biomaterial can 'steal' substrate from its biomaterial-depleted neighbours through diffusive transport.

We remark on two differences in the dispersion curves in Figure 4. First, the simplified, analytically calculated dispersion is larger in magnitude than the numerically one, likely, as previously mentioned, because we overestimated substrate concentration in the simplification. Second, the numerically computed dispersion appears somewhat left-shifted relative to the analytical one. This may be a consequence of an ambiguity in choice of active layer length $\lambda=\sqrt{D / r_{0} \theta_{0}(t)}$ due to the time dependence of $\theta_{0}$ and the fact that, in the simplified model, only $c(H)$ matters. We chose, in formula (4.4), $\theta_{0}=\theta_{0}(0)=0.05$. As $\theta_{0}(t)$ increases in time (in the numerical computation), the instantaneous active layer length in fact decreases in time, effectively left-shifting the numerical dispersion relation relative to the analytical one.

\section{Application: Highly heterogeneous biofilm-host systems}

Having noted length scale formation via linearization and perturbation of homogeneous states, we turn to the full, non-linear behaviour of heterogeneous solutions of the model equations (2.1)-(2.2). Our particular focus is on highly heterogeneous systems, such as can be seen in host-biofilm interactions where microbes are often observed to exist in apparently discrete microcolonies embedded within host matrix [10]. Heterogeneity effects can impact on transport efficiency [5], which in turn can have clinical effects [23,36]. Equations (2.1)-(2.2) are suited as a model for such hybrid systems, but their highly heterogeneous character, for example, related to presence of interior microcolonies including the possibility of rapid material spatial transitions, requires numerical computation.

See Figure 5 for a typical computation, using $f(\theta)=\theta(1-\theta)$ (and $\left.\theta\right|_{t=0}=0.15$ ). Here we have randomly seeded a background 'host' matrix with relatively densely embedded biofilm microcolonies, as in Figure 1, with length scale consistent with the results of the linear theory, that is, colonies separated by roughly the active layer length scale. Top row shows biomaterial volume fraction at a time $t>0$. For reference, the bottom row of Figure 5 shows substrate concentration, demonstrating its depletion.

Note that both vertical active layer formation and internal (to microcolonies) active layers show similar observations in in-vivo biofilm infections [10]. In particular, computations suggest that microcolonies nearest the substrate source become hotspots of activity, capable of forming internal active layers. This is rather different than the homogenous case and this local active layer formation may have consequences for function as well as tolerance to host defense $[38,42]$. Relatively inactive biomaterial, hidden by an active layer, is more tolerant to antimicrobial attacks of various forms. That this defense mechanism may occur globally as well as at the microcolony level (even in regions with substantial substrate penetration) is potentially relevant to understanding the recalcitrance of chronic host-biofilm infections.

For comparison, we also show a computation with a relatively sparse embedding of microcolonies, see Figure 6. Note that in contrast to the more densely populated examples in Figure 5 that microcolonies grow uniformly and at approximately the same rate and there is no emergent active layer either within microcolonies or at a larger active layer 

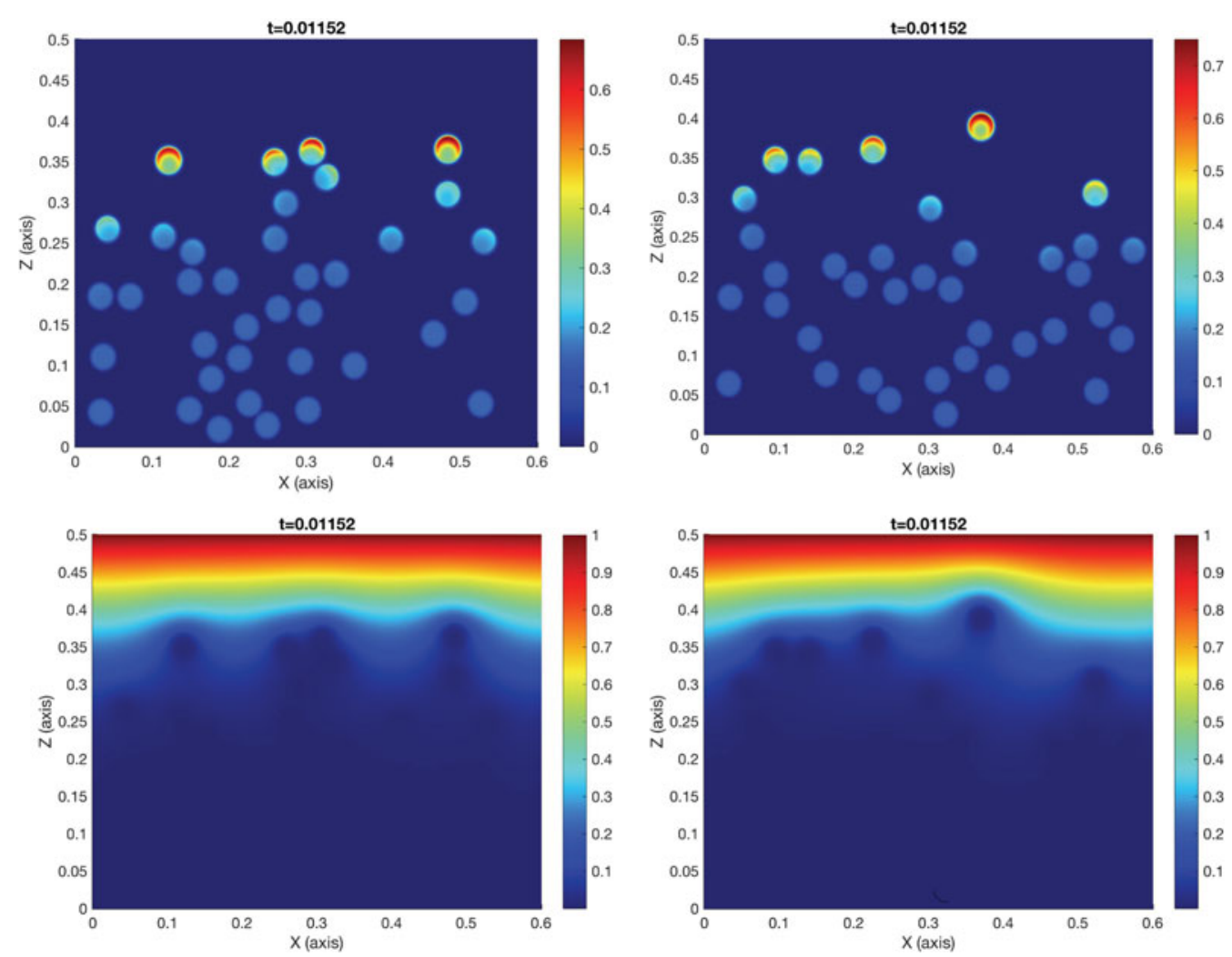

FIGURE 5. Two realisations of randomly embedded circular microcolonies. Top: biomaterial volume fraction. Bottom: substrate concentration.

scale as was seen in Figure 5. Hence, microbes are at approximately uniform activity level, potentially reducing diversity of response to external challenge.

A number of models have addressed diffusive transport limitation as a component of observed persistence of microbial infections as well as possible treatment strategies in response, for example, $[1,13,14,20,21,40,41]$. However, these models assume homogenized biofilm, and hence, do not account for the sort of internal active (and inactive) layers that occur in microcolonies, at least as suggested in the presented model as in Figure 5, which would only aggravate the problem of disinfection.

\section{Computational methods}

Computations use high-order discontinuous Galerkin (DG) finite-element (FE) numerical schemes. Since finite elements generate a variational formulation of system (2.1)-(2.2), the effects of sharp transitions can be handled using high-order numerical quadratures in conjunction with compatible meshes. We employ mesh adaptivity, when required, to resolve the effect of microcolonies on oxygen profiles [4,5], see for example Figure 7. High-order approximations can be obtained by using higher degree basis functions. Another attractive feature of the DG method here is the ease with which it accounts for various types of 

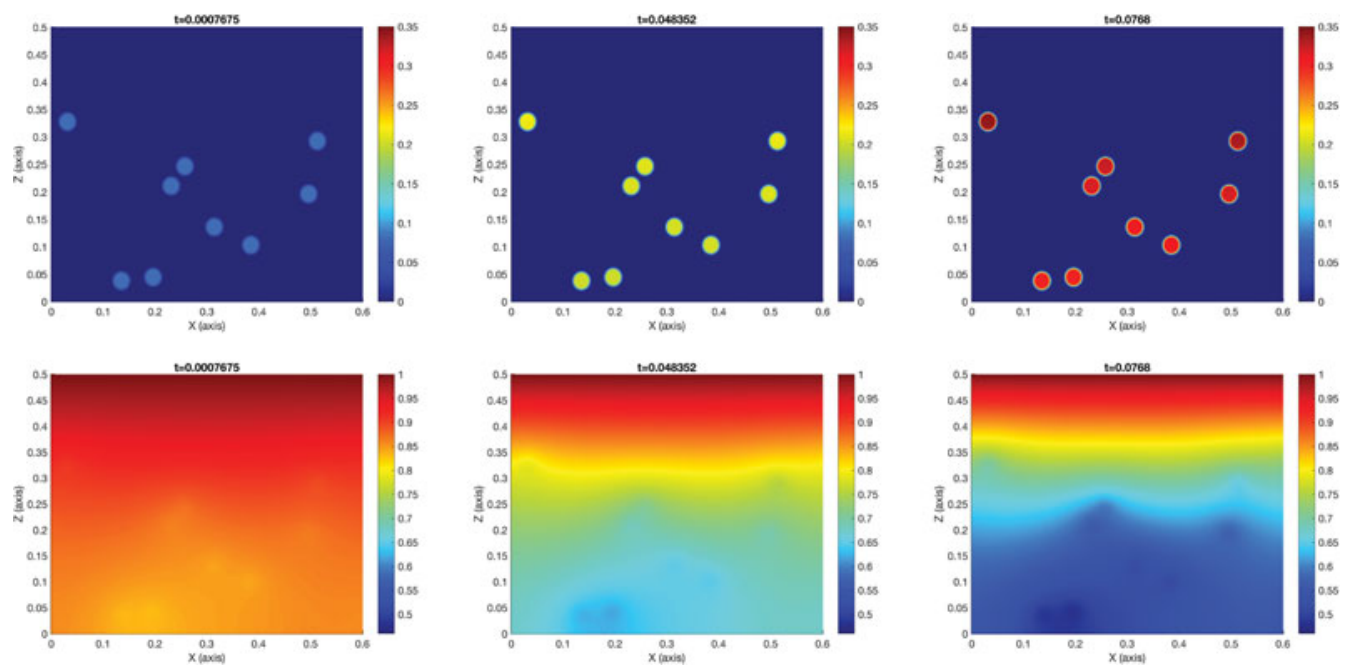

FIGURE 6. Time snapshots from a realisation of randomly but relatively sparsely embedded circular microcolonies. Top: biomaterial volume fraction at three successive times. Bottom: substrate concentration at the same three times.
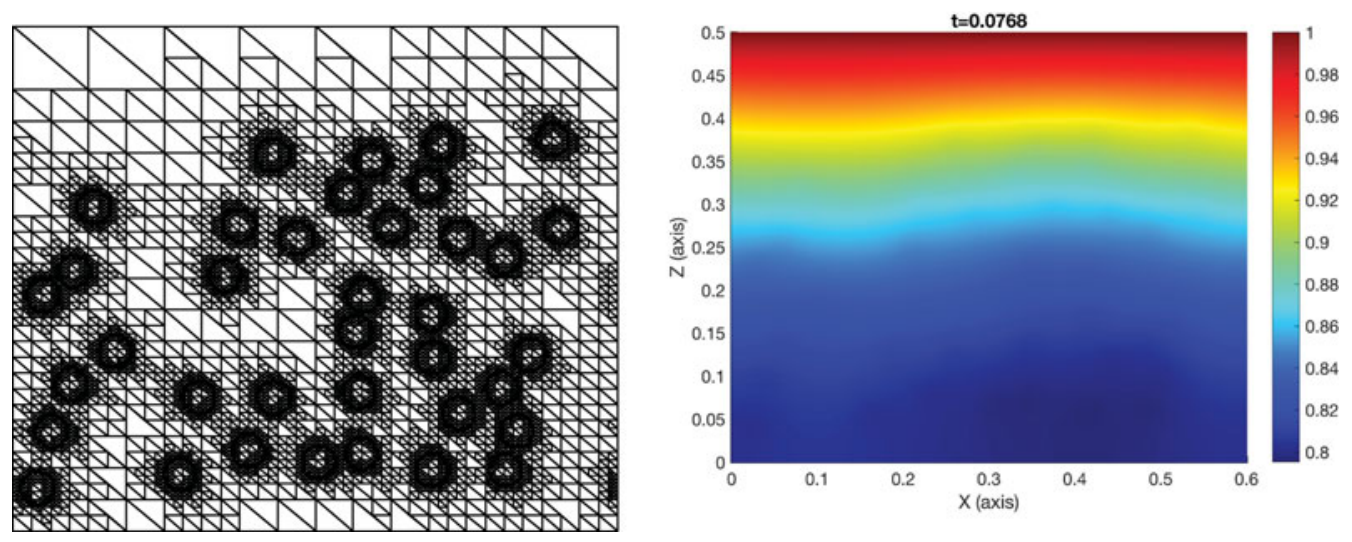

FIGURE 7. (Left) An example of a typical 1-irregular (i.e., at most one hanging node per element edge) adaptive mesh. Use of hanging nodes minimises mesh propagation. The mesh is obtained by successive refinement (using quadrisection) and coarsening of an initial mesh containing only a few large triangles. The triangles are selected to be refined or coarsened according to a marking strategy described in [3] and references therein, based on inverse inequality estimates. The same number of boundary edges occur on opposing periodic domain sides as a result of the periodic mapping used. (Right) Computed substrate concentration using the same mesh.

boundary conditions by incorporating them into the weak formulation. A comprehensive review of DG methods for the spatial discretisation of steady and unsteady PDEs can be found in the literature $[7,17,34]$.

Periodic boundary conditions (in the horizontal direction) for the nutrient equation (2.1) are implemented weakly, following the ideas in [43], for the Poisson equation. This is consistent with the classic DG formulations and the principal behind DG methods. 
The method proposed in [43] requires the existence of the same number of edges in corresponding periodic boundaries and suggests the creation of a map between matching periodic edges, defined as periodic pairs $e_{p}$, that is, $e_{p}:=\left\{e_{i}, e_{j}\right\}$, where $e_{i} \subseteq\{x=0\} \times[0, L]$ and $e_{j} \subseteq\{x=P\} \times[0, L],(P$ is the length of the periodic domain). Essentially, a periodic pair is treated as a single interior edge and the corresponding extra terms are introduced in the bilinear forms. When dealing with adaptive meshes, it is also necessary to correctly maintain the periodic map due to the fact that refined periodic edges may create an imbalance in the number of edges on each periodic boundary [6].

For time-stepping, the $\Theta$-method is employed - the choice of $\Theta=0.5$ corresponds to the Crank-Nicholson method, which provides in theory second-order accuracy in time. We decouple equations (2.1)-(2.2) using a time relaxation. Thus, they can be solved sequentially, resulting in the following fully discrete scheme: find $\left(\theta_{h}, c_{h}\right) \in V_{h}^{q}$, such that

$$
\begin{aligned}
\left(\theta_{h}{ }^{n}-\theta_{h}{ }^{n-1}, w\right) & =D t \times\left\{\Theta\left(f\left(\theta_{h}^{n}\right) r\left(c_{h}^{n}\right), w\right)+\left((1-\Theta) f\left(\theta_{h}^{n-1}\right) r\left(c_{h}^{n-1}\right), w\right)\right\}, \\
D_{n} \mathcal{B}_{h}^{n}\left(c_{h}{ }^{n}, w\right) & =-\left(\theta_{h}^{n-1} r\left(c_{h}^{n}\right), w\right),
\end{aligned}
$$

where $w \in V_{h}^{q}$ is a DG basis function and $V_{h}^{q}$ is the DG finite dimensional space [7], consisting of piecewise polynomial elements of degree $q$. Recall $f(\theta)=\theta(1-\theta)$ or $f(\theta)=\theta$. The usual $L^{2}$-inner product is denoted by $(\cdot, \cdot)$ and $\mathcal{B}_{h}^{n}(\cdot, \cdot)$ is the DG bilinear form corresponding to the Laplacian operator in variational form with the prescribed BCs [34,43]. The $q$ th-order DG basis polynomials provide $O\left(h^{q+1}\right)$ accuracy in the $L^{2}$-norm of the spatial error at each time step, with $h$ being the mesh discretisation parameter. Here, we use cubic polynomials which, in theory, gives us an $L^{2}$-spatial error that is $O\left(h^{4}\right)$. We employ fixed point iteration with extrapolation to solve the nonlinear systems. Artificial time is used for (6.2) when nonlinear iteration alone was too slow. Multilevel solvers tailored to our adaptive multilevel mesh are utilised to solve the linearised algebraic system arising from the numerical discretisation. A conjugate gradient solver with multigrid $[2,11,12]$ preconditioning is employed. Use of the symmetric interior penalty variant of DG-FE [7] results in symmetric positive-definite mass and stiffness matrices that have block structure benefiting the design of our iterative solver and preconditioner.

\section{Discussion}

Microbial communities are also physical structures, and as such, are subject to physical constraints. In particular, in many cases, community function is significantly impacted by diffusive transport. And if community function is effected, then so is community form. One possible form of such impact is fingering. Here, we propose another possible example in the form of a sort of internal version of fingering in which internal, local activity hot spots are able to enhance diffusive flux and thus amplify. As such, a characteristic length scale is suggested, namely that of the active length. On this and shorter scales, diffusion effectively mixes the local concentration gradient and hence reaction-driven depletion can be ameliorated.

This observation leads to the prediction that microbial systems, including host-microbe systems, may exhibit heterogeneous microcolony hotspots with length scales determined by 
diffusive tranport limitation. Such microcolonies, as can be seen in Figure 5, can interact to deplete substrate (such as oxygen) but can also have their own internal, diffusionlimited structures. The resulting structure, consequent to the presence of diffusion limited transport, can have importance in microbial function and in host-microbe interactions.

Our approximated linear theory, supported by numerics, does not directly distinguish a preferred structure wavelength; instead, roughly, all wavelengths below the active length are equally favored. This is a symptom of model simplicity. Some additional mechanism could be added in order to cut off short wavelengths depending on the circumstance. For example, in a host-biofilm system, smaller structures may be more susceptible to host defense than larger ones [10]. In our simulations, numerical diffusivity creates an effective lower bound. Of course, structures smaller than a single cell cannot occur in reality anyway so there is always a cutoff at some point.

We also do not include biomaterial mobility. For example, microcolonies do not spread through growth. Such effects have been included in other models, including other multidimensional continuum-based models $[15,18,19]$. Accounting for displacement, growth-driven or otherwise, introduces complications which might serve to obscure our main point regarding diffusion limitation. A conseqence, though, is that we do not predict influence of diffusion on microcolony size, likely an important statistic.

\section{Acknowledgement}

The authors thank P. S. Stewart for comments and suggestions.

\section{References}

[1] Anguige, K., King, J. R. \& Ward, J. P. (2005) Modelling antibiotic- and quorum sensing treatment of a spatially-structured Pseudomonas aeruginosa population. J. Math. Biol. 51, 557-594.

[2] Aristotelous, A. C., Karakashian, O. A. \& Wise, S. M. (2013) A mixed discontinuous Galerkin, convex splitting scheme for a modified Cahn-Hilliard equation and an efficient non-linear multigrid solver. DCDS-B 18, 2211-2238.

[3] Aristotelous, A. C., Karakashian, O. A. \& Wise, S. M. (2015) Adaptive, second-order in time, primitive-variable discontinuous Galerkin schemes for a Cahn-Hilliard equation with a mass source. IMA J. Numer. Anal. 35, 1167-1198.

[4] Aristotelous, A. C. \& Haider, M. A. (2014) Use of hybrid discrete cellular models for identification of macroscopic nutrient loss in reaction-diffusion models of tissues. Int. J. Numer. Method Biomed. Eng. 30, 767-780.

[5] Aristotelous, A. C., Klapper, I., Grabovsky, Y., Pabst, B., Pitts, B. \& Stewart, P. S. (2015) Diffusive transport through a model host-biofilm system. Phys. Rev. E 92, 022703.

[6] Aristotelous, A. C. \& Papanicolaou, N. C. (2016) A discontinuous Galerkin method for unsteady two-dimensional convective flows. In: American Institute of Physics (AIP) Conference Proceedings, Varna, Bulgaria, Vol. 1773, 110002.

[7] Arnold, D. N., Brezzi, F., Cockburn, B. \& Marini, L. D. (2002) Unified analysis of discontinuous Galerkin methods for elliptic problems. SIAM J. Numer. Anal. 39, 1749-1779.

[8] Bernstein, H. C., Beam, J. P., Kozubal, M. A., Carlson, R. P. \& Inskeep, W. P. (2013) In situ analysis of oxygen consumption and diffusive transport in high-temperature acidic iron-oxide microbial mats. Environ. Microbiol. 15, 2360-2370. 
[9] Buarnsholt, T., Jensen, P. Ø., Fiandaca, M. J., Pedersen, J., Hansen, C. R., Andersen, C. B., Pressler, T., Givskov, M. \& Høiby, N. (2009) Pseudomonas aeruginosa biofilms in the respiratory tract of cystic fibrosis patients. Pediatr. Pulmonol. 44, 547-558.

[10] Buarnsholt, T., Alhede, M., Alhede, M., Eickhardt-Sørensen, S. R., Moser, C., Kühl, M., Jensen, Ø. P. \& HøıвY, N. (2013) The in vivo biofilm. Trends Microbiol. 21, 466-474.

[11] Bramble, J. H. (2003) Multigrid Methods, Research Notes in Mathematics Series, Chapman and Hall/CRC, London.

[12] Brenner, S. C. \& Sung, L. Y. (2006) Multigrid algorithms for C0 interior penalty methods. SIAM J. Numer. Anal. 44, 199-223.

[13] Cogan, N. C. (2008) Two-fluid model of biofilm disinfection. Bull. Math. Biol. 70, 800-819.

[14] Cogan, N. G., Cortez, R. \& Fauci, L. (2005) Modeling physiological resistance in bacterial biofilms. Bull. Math. Biol. 67 831-853.

[15] Cogan, N. G. \& Keener, J. P. (2004) The role of the biofilm matrix in structural development. Math. Med. Biol. 21, 147-166.

[16] Coufort, C., Derlon, N., Ochoa-Chaves, J., Line, A. \& Paul, E. (2007) Cohesion and detachment in biofilm systems for different electron acceptor and donors. Water Sci. Technol. 55, 421-428.

[17] Di Pietro, D. A. \& Ern, A. (2012) Mathematical Aspects of Discontinuous Galerkin Methods, Springer, Berlin.

[18] Dockery, J. D. \& Klapper, I. (2002) Finger formation in biofilms. SIAM J. Appl. Math. 62, 853-869.

[19] Eberl, H. J., Parker, D. F. \& Van Loosdrecht, M. C. M. (2001) A new deterministic spatio-temporal continuum model for biofilm development. J. Theor. Med. 3, 161-175.

[20] Eberl, H. J. \& Sudarsan, R. (2008) Exposure of biofilms to slow flow fields: The convective contribution to growth and disinfection. J. Theor. Biol. 253, 788-807.

[21] Efendiev, M. A., Demaret, L., Lasser, R. \& Eberl, H. J. (2008) Analysis and simulation of a meso-scale model of diffusive resistance of bacterial biofilms to penetration of antibiotics. Adv. Math. Sci. Appl. 18, 269-304.

[22] Galy, O., Latour-Lambert, P., Zrelli, K., Ghigo, J.-M., Beloin, C. \& Henry, W. (2012) Mapping of bacterial biofilm local mechanics by magnetic microparticle actuation. Biophys. J. 103, 1400-1408.

[23] Hopf, H. W., Hunt, T. K., West, J. M., Blomquist, P., Goodson III, W. H., Jensen, J. A., Jonsson, K., Paty, P. B., Rabkin, J. M., Upton, R. A., von Smitten, R. \& Whitney, J. D. (1997) Wound tissue oxygen tension predicts the risk of wound infection in surgical patients. Arch. Surg. 132, 997-1004.

[24] James, G. A., Zhao, A. G., Usui, M., Underwood, R. A., Nguyen, H., Beyenal, H., Pulcini E.D., Hunt, A. A., Bernstein, H. C., Fleckman, P., Olerud, J., Williamson, K., Franklin, M. J. \& StewART, P. S. (2016) Microsensor and transcriptomic signatures of oxygen depletion in biofilms associated with chronic wounds. Wound Repair Regen. 24, 373-383.

[25] Klapper, I. (2013) Productivity and equilibrium in simple biofilm models. Bull. Math. Biol. 74, 2917-2934.

[26] Klapper, I. \& Dockery, J. (2006) Role of cohesion in the material description of biofilms. Phys. Rev. E 74, 031902.

[27] Klapper, I. \& Dockery, J. (2010) Mathematical description of microbial biofilms. SIAM Rev. 52, 221-265.

[28] Klapper, I., Dockery, J. \& Smith, H. (2014) Niche partitioning along an environmental gradient. SIAM J. Appl. Math. 74, 1511-1534.

[29] Lehner, B. A. E., Schmieden, D. T. \& Meyer, A. S. (2017) A straightforward approach for 3D bacterial printing. ACS Synth. Biol. 6, 1124-1130.

[30] Lewandowski, L. (2000) MIC and biofilm heterogeneity. Proc. Corros., NACE-400, 1-7. 
[31] Mitri, S., Clarke, E. \& Foster, K. R. (2016) Resource limitation drives spatial organization in microbial groups. ISME J. 10, 1471-1482.

[32] Nadell, C. D., Drescher, K. \& Foster, K. R. (2016) Spatial structure, cooperation and competition in biofilms. Nat. Rev. Microbiol. 14, 589-600.

[33] Picioreanu, C., van Loosdrecht, M. C. \& Heijnen, J. J. (2000) Effect of diffusive and convective substrate transport on biofilm structure formation: A two-dimensional modeling study. Biotechnol. Bioeng. 69, 504-515.

[34] Rivière, B. (2008) Discontinuous Galerkin Methods for Solving Elliptic and Parabolic Equations, SIAM, Philadelphia.

[35] Schaffner, M., Rühs, P. A., Coulter, F., Kilcher, S. \& Studart, A. R. (2017) 3D printing of bacteria into functional complex materials. Sci. Adv. 3, eaao6804, DOI: 10.1126/sciadv.aao6804.

[36] Schobert, M. \& Tielen, P. (2010) Contribution of oxygen-limiting conditions to persistent infection of Pseudomonas aeruginosa. Future Microbiol. 5, 603-621.

[37] Sønderholm, Koren, K., Wangpraseurt, D., Jensen, P. Ø., Kolpen, M., Kragh, K. N., BJARnsholt, T. \& KüHL (2018) Tools for studying growth patterns and chemical dynamics of aggregated Pseudomonas aeruginosa exposed to different electron acceptors in an alginate bead model. npj Biofilms Microbiomes 4, art. no. 3.

[38] Stewart, P. S. (2002) Mechanisms of antibiotic resistance in bacterial biofilms. Int. J. Med. Microbiol. 292, 107-113.

[39] Stewart, P. S. \& Franklin, M. J. (2008) Physiological heterogeneity in biofilms. Nat. Rev. Microbiol. 6, 199-210.

[40] Stewart, P. S. \& Raquepas, J. B. (1995) Implications of reaction-diffusion theory for disinfection of microbial biofilms by reactive antimicrobial agents. Chem. Eng. Sci. 50, 3099-3104.

[41] Szomolay, B., Klapper, I. \& Dindos, M. (2010) Analysis of adaptive response to dosing protocols for biofilm control. SIAM J. Appl. Math. 70, 3175-3202.

[42] Szomolay, B., Klapper, I., Dockery, J. \& Stewart, P. S. (2005) Adaptive responses to antimicrobial agents in biofilms. Environ. Microbiol. 7, 1186-1191.

[43] Vemaganti, K. (2007) Discontinuous Galerkin methods for periodic boundary value problems. Num. Methods Partial Differ. Equat. 23, 587-596.

[44] Zhang, Z., Nadezhina, E. \& Wilkinson, K. J. (2011) Quantifying diffusion in a biofilm of Streptococcus mutans. Antimicrob. Agents Chemother. 55, 1075-1081. 